\title{
STABILITY OF ELECTRON ORBITS IN THE STRONG WAKE FIELDS GENERATED BY A TRAIN OF FSEC BUNCHES*
}

\author{
Changbiao Wang ${ }^{1}$, J. L. Hirshfield ${ }^{1,2}$, and T. C. Marshall ${ }^{3}$ \\ ${ }^{1}$ Physics Department, Yale University, New Haven, CT 06520 USA \\ ${ }^{2}$ Omega-P, Inc., New Haven, CT 06520 USA \\ ${ }^{3}$ Applied Physics Department, Columbia University, New York, New York 10027, USA
}

\begin{abstract}
A tall, dielectric-lined rectangular wake field microstructure is being analyzed as a possible stageable element of an advanced linear wake field accelerator, to be driven by a train of fsec microbunches. These microbunches would be chopped out of a longer bunch using a powerful $\mathrm{CO}_{2}$ laser and formed into a rectangularprofile bunch using a quadrupole. The fsec bunches set up a periodic wake field in the microstructure that can be built up to $600 \mathrm{MV} / \mathrm{m}$, for example, using ten 3-fsec bunches each containing $2 \mathrm{pC}$ of charge. Results are described from computations of test particle electron orbits in the longitudinal and transverse wake fields excited by these fsec bunches. It is found that test electrons in drive bunches will be well confined within the structure for a travel distance of $\sim 10 \mathrm{~cm}$ and test electrons located in an accelerated bunch will have stable motion for at least $50 \mathrm{~cm}$.
\end{abstract}

\section{INTRODUCTION}

Acceleration of electrons in wake fields set up by a series of driving bunches in a dielectric structure has shown promise as a linear accelerator in which large gradients might be possible $[1,2]$. Such wake fields are interesting because they do not require power injected into the structure from an external source, but rather use fields set up by bunches obtained from a conventional rf linac. Recently, we have studied the use of tall, planar dielectric wake field structures having micron-scale dimensions [3]. Such structures are capable of precision manufacture using microcircuit technologies, and have the capability of achieving very high field gradients: indeed, a series of ten, 3-fsec 1-pC charge bunches has recently been shown to set up a wake field of $\sim 500 \mathrm{MeV} / \mathrm{m}$ in a structure $18.8 \times 150 \mu \mathrm{m}$ in cross section [4]. The bunches are $10 \mu \mathrm{m}$ wide, and dielectric slabs a few $\mu \mathrm{m}$ thick line the structure. Planar dielectric structures offer the attraction of improving the stability of the bunch motion and the amount of charge carried compared with a cylindrical structure of comparable size, and the small transverse dimension permits a large wakefield to be developed.

The bunches could be obtained initially from a 500 $\mathrm{MeV}$ rf linac-type source, and are processed using a LACARA accelerator "chopper" [3], or possibly an IFEL [5] used as a "pre-buncher", so as to obtain a sequence of bunches a few fsec in duration. A TW $\mathrm{CO}_{2}$ laser is used as a "modulator" [3] of the original psec, $\mathrm{nC}$ bunch

*Work supported by US DoE. provided by the linac to form such a sequence of short bunches, each having charge in the $\mathrm{pC}$ range. These drive bunches, the energy of which can be recycled, would in practice be followed by an accelerated bunch which is situated in the accelerating component of $E_{z}$ which follows the drive bunch train. In this way fields comparable with those achieved in laser plasma wake field accelerators can be set up, yet the energy is obtained largely from the rf linac source rather than the laser. We have found that it is possible to distort the original circular cross section of the input bunches into a rectangular profile, using a quadrupole, and that the rectangular profile is maintained for several centimeters of travel [4].

Transverse fields set up by the bunch have been calculated, and an estimate has been made of how far a drive bunch might travel without additional focusing [6] (several $\mathrm{cm}$ ). Also, studies have been made of fields in 3D using the PIC code KARAT. The $E_{z}$ component of wake field was found to be rather uniform in cross section. In the structure under study, the wake fields are dominated by two modes having nearly the same periodicity (about $21 \mu \mathrm{m}$ in the chosen geometry). In this paper, we study the motion of test particles which are situated initially in a grid of loci at the location of any drive or accelerated bunch (see Fig. 1). Our findings show that adequate stability of the drive bunches can be obtained by choosing a tall structure $(300 \mu \mathrm{m})$, and that a certain group of test particles can be accelerated for a distance of $\sim 1 / 2 \mathrm{~m}$ or more without external focusing, maintaining a nearly stable profile and gaining energy of $300 \mathrm{MeV}$ in that distance.

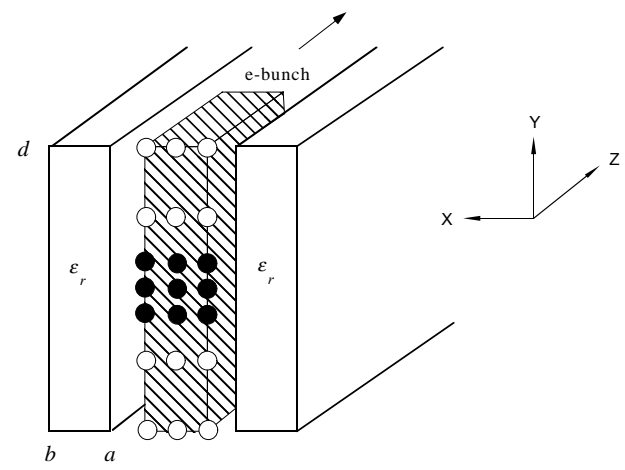

Figure 1: Schematic slab bunch within a planar wake field structure. Circles stand for test particles in the (decelerated) drive bunch while dots stand for test particles in the accelerated bunch. 
NUMERICAL PROCEDURE AND MODEL

A complete theory for excitation of wake fields in 3D planar dielectric-lined waveguides, similar in structure to a theory for cylindrical waveguides [7], has been formulated [6]. Park wrote a code [8] based on his theory [6] to calculate distributions of wake field forces excited by a train of rigid electron bunches moving at a constant velocity. In this paper, the code is used to calculate dynamics for test particles by adding a routine solving the Lorentz force equations, in which the wake field forces are as in Ref. 6.

The dielectric structure is shown in Fig. 1, with the beam channel width $2 a=15 \mu \mathrm{m}$, the dielectric thickness $b-a=1.9 \mu \mathrm{m}$, and the dielectric (channel) height $2 d=300$ $\mu \mathrm{m}$, and the relative dielectric constant $\varepsilon_{r}=3$. The rigid drive bunches are $10 \mu \mathrm{m}$ wide, $300 \mu \mathrm{m}$ high, and $1 \mu \mathrm{m}(3$ fsec) long, each containing $2 \mathrm{pC}$ of charge. The initial velocity of test particles is assumed to be the same as that of drive bunch, which has a relativistic energy factor of $\gamma=1000(510.5 \mathrm{MeV})$. The self-field effect and the reaction on the drive bunch are neglected. The test particles in the drive bunch are initially uniformly distributed within a $10 \times 300-\mu \mathrm{m}$ rectangle while the test particles in the accelerated bunch are located within a $10 \times 40-\mu \mathrm{m}$ rectangle. In simulations, 861 computational particles are used to calculate the interaction between wake fields and test particles.

The place of injection of the accelerated bunches can be optimized. At a place behind the last drive bunch appropriate for acceleration, the transverse wake fields are small while the axial field is large. Since the axial slippage between the wake fields and accelerated particles is quite small, one can estimate what wake field peaks are potentially good for acceleration using only the initial field distributions. Therefore, we first find the possible positions and then do dynamic simulations one by one to obtain the best one favoring stable motion.

\section{MOTION OF DRIVE BUNCH}

Coherence of wake fields from different drive bunches requires a fixed bunch spacing. The first dive bunch is set at $z=1200 \mu \mathrm{m}$ and the $10^{\text {th }}$ drive bunch is at $1009.2 \mu \mathrm{m}$, with a spacing of $21.2 \mu \mathrm{m}$. The stability of the $10^{\text {th }}$ drive bunch is examined by initially setting test particles at $z=$ $1009.2 \mu \mathrm{m}$, where the axial wake field force on the axis is $-210.2 \mathrm{MeV} / \mathrm{m}$, excited by first 9 drive bunches.

Fig. 2a shows the dependence of $F_{x}$ on $y$ at $z=1009.2$ $\mu \mathrm{m}$ for $x=0,1,3,5 \mu \mathrm{m}\left(F_{x}\right.$ is anti-symmetric with respect to $x$ ). It is seen that the magnitude of $F_{x}$ increases nearly linearly with $x$, and $F_{x}$ is focusing or defocusing, depending on $y . F_{x}$ is focusing around $y=-95,-25,25$, and $95 \mu \mathrm{m}$ while defocusing around $y=-135,-60,0,60$, and $135 \mu \mathrm{m}$. Fig. $2 \mathrm{~b}$ shows the distributions of the test particles at $z=1009.2 \mu \mathrm{m}, 4 \mathrm{~cm}, 7 \mathrm{~cm}$, and $10 \mathrm{~cm}$ under the influence of the wake fields. The four $x$-direction focusing locations can be easily identified from the $z=4$ $\mathrm{cm}$ distribution. Because of a strong defocusing force in the region of $115 \mu \mathrm{m}<|y|<150 \mu \mathrm{m}$ (two $y$-ends), particles there get lost to walls (see the $z=10-\mathrm{cm}$ distribution). Fig. 2c shows the dependence of average relativistic energy factor and percentage of surviving particles upon axial distance. All the particles go $\sim 2 \mathrm{~cm}$ without hitting any walls, and finally more than $70 \%$ arrive at $z=10 \mathrm{~cm}$. The particles are decelerated from $\langle\gamma\rangle=1000(510.5 \mathrm{MeV})$ to $955.2(487.6 \mathrm{MeV})$.

Simulations show that $94 \%$ of the test particles at the second drive bunch survive traveling $10 \mathrm{~cm}$ while $77 \%$ at the fifth and $73 \%$ at the eighth.

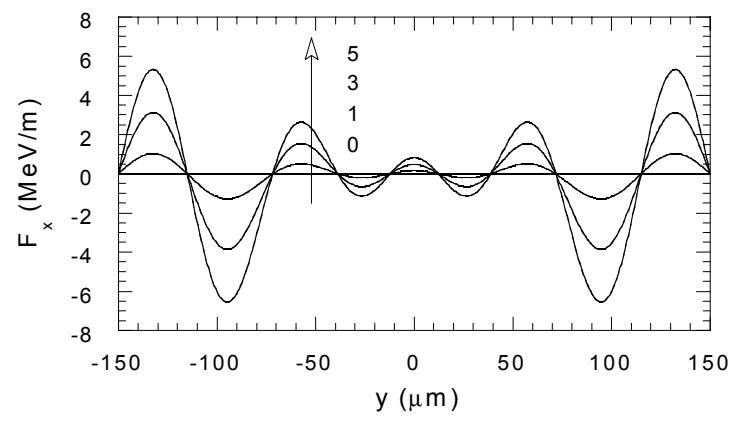

Figure 2a: Dependence of $F_{x}$ on $y$ for $x=0,1,3$, and 5 $\mu \mathrm{m} . \quad F_{x}$ is focusing when $F_{x}<0$, while $F_{x}$ is defocusing when $F_{x}>0$.
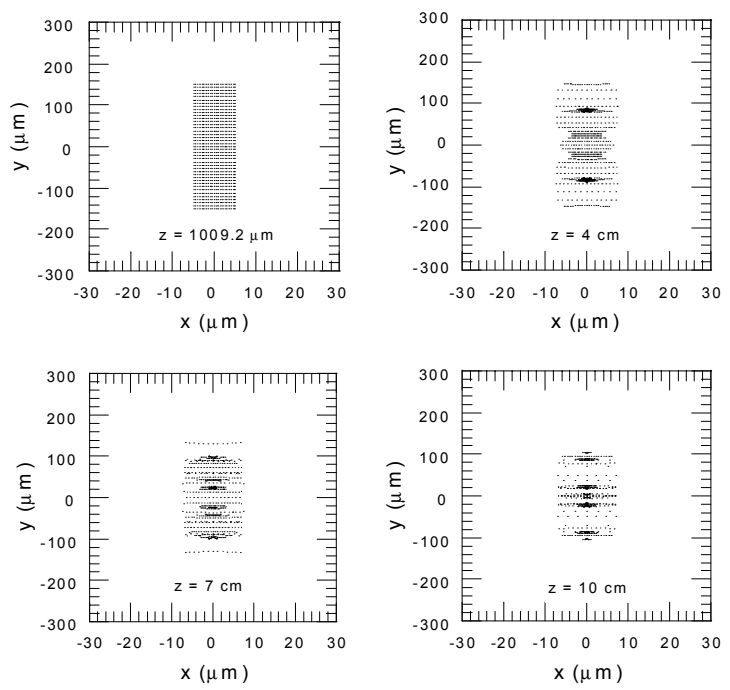

Figure 2b: Drive bunch test particle distributions at $z=$ $1009.2 \mu \mathrm{m}, 4 \mathrm{~cm}, 7 \mathrm{~cm}$, and $10 \mathrm{~cm}$.

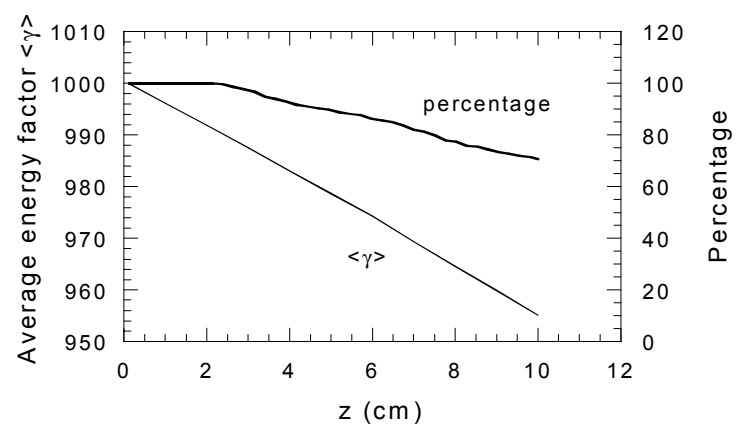

Figure 2c: Dependence of average energy factor and percentage of surviving particles on axial distance. 


\section{MOTION OF ACCELERATED BUNCH}

The accelerated bunch comes from a different source than the drive bunch. The stability of accelerated particles depends on positions in the wake field and the size of the region the particles take up. Simulations show that the test particles in the accelerated bunch following a train of 10 drive bunches should to be situated at $z=60.5 \mu \mathrm{m}$, where the transverse wake field forces are small and focusing in a rectangular region of $|x|<5 \mu \mathrm{m}$ and $|y|<20$ $\mu \mathrm{m}$ while the axial wake field force on the axis reaches its peak, 618.1 MeV/m, as shown in Fig.3a. $F_{x}$ and $F_{y}$ are focusing when less than zero, since they are plotted at $x>$ 0 and $y>0$ respectively.

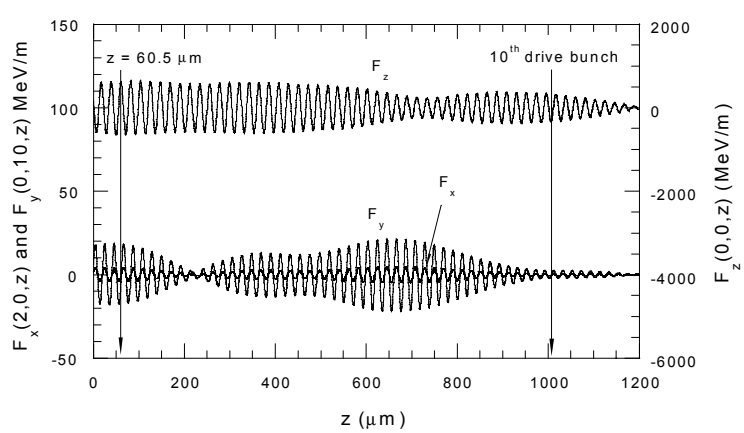

Figure 3a: Dependence of wake field forces $F_{x}(2,0, z)$, $F_{y}(0,10, z)$, and $F_{z}(0,0, z)$ on axial distance $z$, excited by 10 drive bunches. The stability of the accelerated bunch is examined by setting test particles at $z=60.5 \mu \mathrm{m}$.

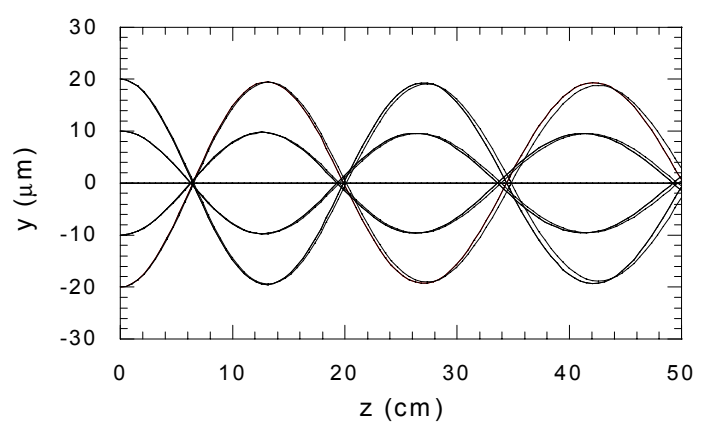

Figure 3b: Orbits on the $y-z$ plane for 15 sampled accelerated particles.
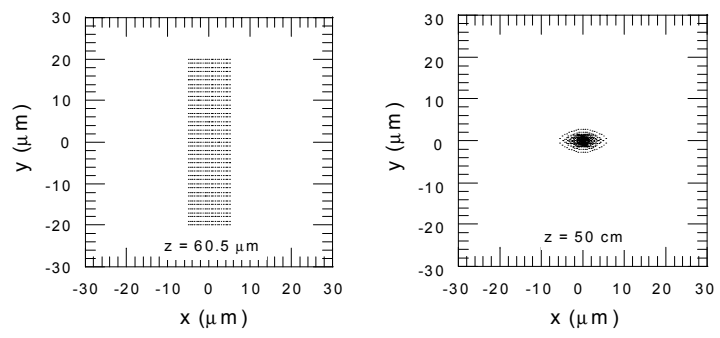

Figure 3c: Test particle distributions on the $x-y$ planes at $z$ $=60.5 \mu \mathrm{m}$ where 861 electrons are initially distributed and at $50 \mathrm{~cm}$ where they are all well focused again.

Simulations show that during the course of acceleration, the particles experience focusing and defocusing alternatively, and they are well confined within a rectangular region of $|x|<7.5 \mu \mathrm{m}$ and $|y|<20 \mu \mathrm{m}$. Fig. $3 \mathrm{~b}$ shows the orbits on the $y-z$ plane for 15 sampled accelerated particles. Fig. $3 \mathrm{c}$ shows the initial distribution $(z=60.5 \mu \mathrm{m})$ and final distribution $(z=50 \mathrm{~cm})$.

It is found that the bunch of particles is accelerated from $\langle\gamma\rangle=1000(510.5 \mathrm{MeV})$ to $1589.1(811.5 \mathrm{MeV})$ in a 50 -cm distance.

\section{DISCUSSION}

In the analysis, the electron bunch is assumed to be cold. A rough estimation of effect of finite emittance can be made as follows. Suppose we take a $500 \mathrm{MeV}$ electron beam with a radius of $50 \mu \mathrm{m}$ and a normalized emittance of $1 \mathrm{~mm}-\mathrm{mrad}$ as an example. The maximum normalized transverse velocity is $2 \times 10^{-5}$ and the maximum transverse displacement caused by the emittance is $7.5 \mu \mathrm{m}$ after a $37.5-\mathrm{cm}$ travel. From this it follows that the emittance effect is not so important for a $10-\mathrm{cm}$ interaction between decelerated test particles and the wake fields. For accelerated test particles, the emittance effect will be reduced because both $F_{x}$ and $F_{y}$ are nearly linearly focusing forces in the whole region the particles take up. Of course, a more sophisticated code is needed to better understand emittance effect on the stability.

We have also studied a 1-pC bunch $150 \mu \mathrm{m}$ tall (half the height and charge as the example) inside the same structure, and find nearly the same acceleration gradient and similar stability behavior.

We have shown that both drive and accelerated bunches can enjoy comparatively stable motion whilst traversing many centimeters through the structure, permitting a very large gradient to be exploited for particle acceleration.

\section{REFERENCES}

[1] T.-B. Zhang, J.L. Hirshfield, T.C. Marshall, and B. Hafezi, Phys. Rev. E56 , (1997) 4647.

[2] J.G. Power, M.E. Conde, W. Gai, R. Konecny, P. Schoessow, and A.D. Kanareykin, Phys. Rev. ST Accel. Beams 3, (2000) 101302.

[3] T.C. Marshall, C. Wang, and J.L. Hirshfield, Phys. Rev. ST Accel. Beams 4, (2002) 121301.

[4] T. C. Marshall, J.-M. Fang, J. L. Hirshfield, C. Wang, V. P. Tarakanov, and S. Y. Park, "Wake Fields Excited in a Micron-Scale Dielectric Rectangular Structure by a Train of Femtosecond Bunches," in Advanced Accelerator Concepts: tenth Workshop, edited by C. E. Clayton and P. Muggli, AIP Conf. Proc. No. 647 (AIP, New York, 2002), p. 361.

[5] Y. Liu, X. J. Wang, D. B. Cline, M. Babzien, J. M. Fang, J. Gallardo, K. Kusche, I. Pogorelsky, J. Skaritka, and A. van Steenbergen, Phys. Rev. Lett. 80, (1998) 4418.

[6] S-Y. Park, C. Wang, and J.L. Hirshfield, "Theory for Wake Fields and Bunch Stability in Planar Dielectric Structures," in Ref. [4], p. 527.

[7] S-Y. Park and J.L. Hirshfield, Phys. Rev. E62, (2000) 1266.

[8] S-Y. Park, Private communications. 